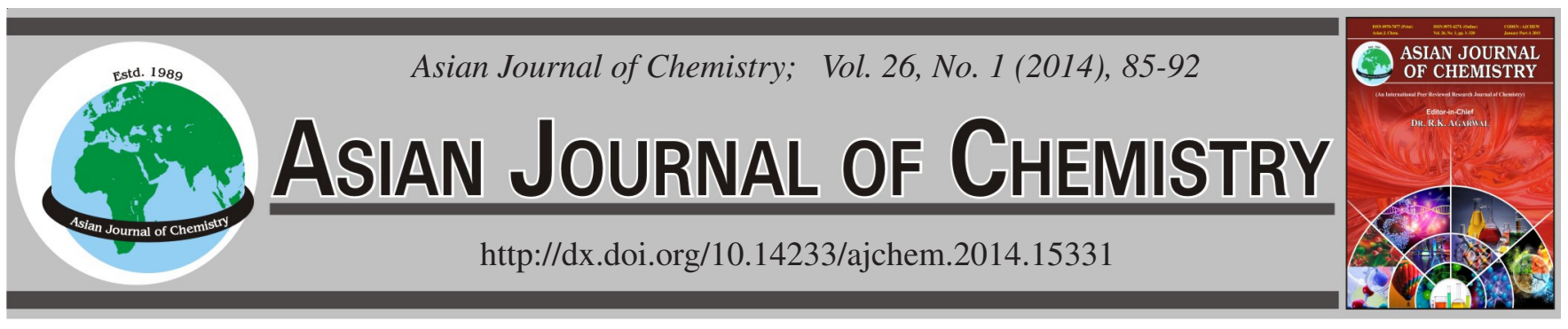

\title{
Synthesis, Characterization, Effect of Triaryl Ring Substituents Groups on Thermal and Spectral Properties of New Soluble Triphenylamine-Based Aromatic Polyamides
}

\author{
Azhar Kamil Rashid ${ }^{1,2, *}$, Rosiya Binti Yahya ${ }^{1}$ and Phang Sook Wai ${ }^{1}$
}

${ }^{1}$ Department of Chemistry, Faculty of Science, University of Malaya, 50603 Kuala Lumpur, Malaysia

${ }^{2}$ University of Baghdad, Baghdad, Iraq

*Corresponding author: Fax: +60 79674193; Tel: +60 104259265; E-mail: azhar_almasody@yahoo.co.uk

\begin{abstract}
Three new triphenylamine-containing aromatic diacid monomers, 4,4'-dicarboxy-4'"-isopropyl-triphenylamine (Ma), 4,4'-dicarboxy-2'4"'dimethyl-triphenylamine (Mb, 4,4'-dicarboxy-4'"-ethyltriphenylamine (Mc) were successfully synthesized via the aromatic nucleophilic fluoro-displacement reaction of 4-fluorobenzonitrile with aniline-derivatives using sodium hydride as the base, followed by alkaline hydrolysis of the dinitrile intermediates (Ia), (Ib), (Ic). A series of poly(amine-amide)s were prepared by the direct phosphorylation polycondensation from the newly synthesized diacid monomers with various aromatic diamines. FTIR, ${ }^{1} \mathrm{H}$ and ${ }^{13} \mathrm{C}$ NMR spectroscopic techniques were used to identify the chemical structures of the dicyano intermediates, the carboxylic acid monomer and the resultant poly(amine-amide)s. These aromatic poly(amine-amide)s were found to be readily soluble in a variety of organic solvents and could afford strong and tough films via solution casting. They exhibited excellent thermal stability associated with high glass transition temperatures $\left(\mathrm{T}_{\mathrm{g}}=248.32-290.46^{\circ} \mathrm{C}\right)$ and $10 \%$ weight loss temperatures in excess of $522^{\circ} \mathrm{C}$ in nitrogen. In dilute $\mathrm{N}$-methyl pyrrolidone solution, these polymers exhibited a medium to strong photoluminescence in the blue region at (425-443) nm. Cyclic voltammetry of the poly(amineamide)s films cast onto an ITO-coated glass substrate in dry acetonitrile containing $0.1 \mathrm{M}$ of tetrabutylammonium perchlorate (TBAP) as an electrolyte exhibited one oxidation redox couples ( $\mathrm{E}_{\text {onset }}$ ) at (1.30-1.37) $\mathrm{V}$ versus $\mathrm{Ag} / \mathrm{AgCl}$ and revealed electrochromic characteristics with a colour change from pale yellow to blue at applied potentials switched between 0.0 and $1.6 \mathrm{~V}$.
\end{abstract}

Keywords: Hole-transporting materials, Triphenylamine, Emitting layer.

\section{INTRODUCTION}

The field of organic light-emitting devices (OLEDs) has attracted considerable interests due to their potential applications in flat panel displays. Materials with hole-transporting capability have been widely used in polymer light-emitting devices. They have relatively high mobility's and low ionization potentials ${ }^{1-6}$ and are easily oxidized to form stable radical cations $^{7}$. The use of conjugated polymers in polymer lightemitting devices (PLEDs), has received a great deal of concern in industry because of several appealing advantages and the most efficient devices using PLEDs is architecture with a thinfilm multilayer structure consists of a hole-transporting layer, an emitting layer and an electron transporting layer sandwiched between two electrodes ${ }^{8}$. Charge carriers (holes and electrons) are injected separately from anode and cathode, recombine in the emitting layer and thus emit light ${ }^{9}$. Many emitting materials have been designed and used in light-emitting devices. However, high-performance light emitting ones are rare because of the intrinsic wide band-gap required for such materials. Many hole transporting materials have been tried for light-emission ${ }^{10}$. Most of the hole-transporting materials which contain amine functionalities show lack sufficient emission characteristics due to reductive quenching ${ }^{11}$. Aromatic polyamides are well accepted as high-performance polymeric materials for their excellent mechanical properties, high thermal stability and good chemical resistance ${ }^{12}$. They are also known as difficult processing materials because of high melting or glass transition temperatures and limited solubility in most organic solvents. To overcome these limitations, polymer-structure modification becomes necessary such as introduction of bulky, packingdisruptive groups into the polymer backbone ${ }^{13-16}$. It has been demonstrated that aromatic polyamides containing threedimensional, propeller-shaped triphenylamine unit had good solubility inorganic solvents while retaining high thermal stability ${ }^{17-19}$. Furthermore, triphenylamine-based polymers are widely used as the hole-transport layer in emitting layerdevices, but they also show interesting electrochromic behaviour $^{20-25}$. The most common method for the preparation of aromatic polyamides is the reaction of diacid compounds 
with diamines at high temperatures. The solvents used are polar aprotic solvents like N,N-dimethyl formamide (DMF), N,Ndimethylacetamide (DMAc), N-methyl pyrrolidone (NMP) and HMPA. Salts, such as $\mathrm{LiCl}, \mathrm{CaCl}_{2}$ or a mixture of both, are often used as solubility promoters because the cations interact with the amide groups, diminishing the strength of the interchain hydrogen bonds. The method was developed by Yamazaki et ll. $^{26}$. In this paper we synthesize new poly(amineamide)s via phosphorylation polycondensation reaction to form newly synthesized monomers. The monomers and polymers characterized structurally by FTIR, ${ }^{1} \mathrm{H}$ and ${ }^{13} \mathrm{C}$ NMR spectroscopic techniques. General properties, thermal, spectral, redox were measured for these poly(amine-amide)s.

\section{EXPERIMENTAL}

4-Isopropylaniline (Merck, $99.8 \%$ ), 2,4-dimethylaniline (Acros, 98.7), 2-ethylaniline (Merck, 99.5), 4-fluorobenzonitrile (Acros, $98.7 \%$ ), sodium hydride $(\mathrm{NaH})$ (Fluka, $60 \%$ ), N,N-dimethylformamide (DMF) (Acros, $99.6 \%$ ), potassium hydroxide (Fluka, $99.8 \%$ ), ethanol (Merck, $99.7 \%$ ), acetic acid (Merck, $98 \%$ ), hydrochloric acid (Merck, $36 \%$ ), dimethyl sulfoxide- $d_{6}$, (DMSO- $d_{6}$ ) (Merck, $99.8 \%$ ), chloroform- $\mathrm{D}_{1},\left(\mathrm{CDCl}_{3}\right)$ (Merck, $\left.99.8 \%\right)$, P-phenylenediamine (Merck, $98 \%$ ), 1,5-diaminonaphthalene (Fluka, 98.8\%), pyridine (Merck, $97.8 \%$ ), triphenylphosphite (Fluka, 99\%), calcium chloride (Merck, 99,6\%), N-methyl pyrolidone (NMP) (Fluka, 99.6\%) were used without further purification.

The FT-IR spectrum of the synthesizied compounds were recorded with a Spotlight 400 Perkin Elmer spectrometer. ${ }^{1} \mathrm{H}$ NMR (400 MHz) and ${ }^{13} \mathrm{C}$ NMR (100 MHz) measurements were performed with a JEOL spectrometer. UV-visible absorption and photoluminescence spectra were recorded in $\mathrm{N}$ methyl pyrolidone with concentration $\left(10^{-5} \mathrm{M}\right)$ on a Cary 60 UV-visible spectrophotometer. Thermal data were obtained from a Perkin Elmer DSC6 instrument. The data were recorded under nitrogen atmosphere at a heating rate of $20^{\circ} \mathrm{C} / \mathrm{min}$ with a SDT Q600 thermogravimetric analyser. The thermal decomposition data were recorded at a heating rate of $20^{\circ} \mathrm{C} / \mathrm{min}$ under nitrogen atmosphere with a SDT Q600 thermogravimetric analyser (TGA Instrument).

Monomer synthesis: The monomers (Ma-Mc) have synthesized via the route as explained in the Scheme-I.
Synthesis of 4,4'-dicyano-4"'-isopropyltriphenylamine (Ia): A mixture of $1.40 \mathrm{~g}(0.02 \mathrm{~mol})$ of sodium hydride and $80 \mathrm{~mL}$ of DMF was stirred at room temperature for $0.5 \mathrm{~h}$. To the mixture, $2.70 \mathrm{~g}(0.02 \mathrm{~mol})$ of 4-isopropylaniline and 4.84 $\mathrm{g}(0.02 \mathrm{~mol})$ of 4 -fluorobenzonitrile were added in sequence. The mixture was heated with stirring at $120^{\circ} \mathrm{C}$ for $24 \mathrm{~h}$ under nitrogen and then precipitated into $150 \mathrm{~mL}$ of cold water ${ }^{27,28}$. The products were filtered and recrystallized from ethanol to give pale yellowish solid 3.43 g (yield: $51 \%)$, m.p. $=(185-$ 188) ${ }^{\circ} \mathrm{C}$. FTIR: $2211.4 \mathrm{~cm}^{-1}(\mathrm{C} \equiv \mathrm{N}) .{ }^{1} \mathrm{H} \mathrm{NMR}\left(400 \mathrm{MHz}, \mathrm{CDCl}_{3^{-}}\right.$ d, $\delta$ ppm):7, 42 (d, 4H, $\left.\mathrm{H}_{\mathrm{d}}\right), 7.15(\mathrm{~d}, 2 \mathrm{H}, \mathrm{Ha}), 7.03(\mathrm{~d}, 4 \mathrm{H}$, $\mathrm{Hc}), 6.97\left(\mathrm{~d}, 2 \mathrm{H}, \mathrm{H}_{\mathrm{b}}\right), 2.85\left(\mathrm{~m}, 1 \mathrm{H}, \mathrm{H}_{\mathrm{CH}}\right), 1.20\left(\mathrm{~d}, 6 \mathrm{H}, \mathrm{H}_{\mathrm{CH} 3}\right)$. ${ }^{13} \mathrm{C}$ NMR (400 MHz, $\left.\mathrm{CDCl}_{3}-\mathrm{d}, \delta \mathrm{ppm}\right): 150.20\left(\mathrm{C}_{5}\right)$, 147.47( $\left.\mathrm{C}_{4}\right), 105.38\left(\mathrm{C}_{8}\right), 118.92(\mathrm{CN}),, 122.54\left(\mathrm{C}_{6}\right), 126.93\left(\mathrm{C}_{2}\right)$, $128.21\left(\mathrm{C}_{3}\right), 133.40\left(\mathrm{C}_{7}\right), 142.38\left(\mathrm{C}_{1}\right), .33 .62\left(\mathrm{C}_{\mathrm{CH}}\right), 23.84\left(\mathrm{C}_{\mathrm{CH}_{3}}\right)$.

4,4'-Dicarboxy-4"-isopropyltriphenylamine (Ma): A mixture of $15.5 \mathrm{~g}$ of potassium hydroxide and $6.74 \mathrm{~g}(0.02$ mol) of the dinitrile compound (1) in $60 \mathrm{~mL}$ of ethanol and 60 $\mathrm{mL}$ of distilled water was stirred at $100{ }^{\circ} \mathrm{C}$ until no further ammonia was generated. The time taken to reach this stage was $c a .24 \mathrm{~h}$. The solution was cooled and the $\mathrm{pH}$ value was adjusted by dilute $\mathrm{HCl}$ to near 3 . The yellowish precipitate formed was collected by filtration, washed thoroughly with water. Recrystallization from acetic acid gave pale yellow crystals $6.0 \mathrm{~g}$ (80\% yield); m.p. $=268-270{ }^{\circ} \mathrm{C}$. FTIR: 1681 $\mathrm{cm}^{-1}(\mathrm{C}=\mathrm{O}), 2987 \mathrm{~cm}^{-1}(\mathrm{O}-\mathrm{H}) .{ }^{1} \mathrm{H}$ NMR (400 MHz, DMSO$\left.d_{6}, \delta \mathrm{ppm}\right): 7.99\left(\mathrm{~d}, 4 \mathrm{H}, \mathrm{H}_{\mathrm{d}}\right), 7.28\left(\mathrm{~d}, 2 \mathrm{H}, \mathrm{H}_{\mathrm{a}}\right), 7.07\left(\mathrm{~d}, 2 \mathrm{H},\left(\mathrm{H}_{\mathrm{b}}\right)\right.$, $7.00\left(\mathrm{~d}, 4 \mathrm{H}, \mathrm{H}_{\mathrm{C}}\right), 2.88\left(\mathrm{~m}, 1 \mathrm{H}, \mathrm{H}_{\mathrm{CH}}\right), 1.21\left(\mathrm{~d}, 6 \mathrm{H}, \mathrm{H}_{\mathrm{CH}_{3}}\right), 12.70$ (br, 2H, COOH) $.^{13} \mathrm{C} \mathrm{NMR} \mathrm{(400MHz,DMSO-} d_{6}, \delta$ ppm): 166.83 $(\mathrm{COOH}), 150.44\left(\mathrm{C}_{5}\right), 145.82\left(\mathrm{C}_{4}\right), 143.22\left(\mathrm{C}_{1}\right), 130.97\left(\mathrm{C}_{7}\right)$, $128.01\left(\mathrm{C}_{3}\right), 124.42\left(\mathrm{C}_{2}\right) 126.56\left(\mathrm{C}_{8}\right), 121.86\left(\mathrm{C}_{6}\right), 23.81(\mathrm{CH})$, $23.81\left(\mathrm{CH}_{3}\right)$.

4,4'-Dicyano-4"'-2,4-dimethylaniline(Ib): The synthesis of this compound was the same to that for Ia. The product was filtered and recrystallized from ethanol to give pale yellowish solid 2.31 g (yield: $71.5 \%$ ), m.p. $=146-148^{\circ} \mathrm{C}$. FTIR: 2222.4 $\mathrm{cm}^{-1}(\mathrm{CN}) .{ }^{1} \mathrm{H} \mathrm{NMR}\left(400 \mathrm{MHz}, \mathrm{CDCl}_{3}-d_{1}, \delta \mathrm{ppm}\right):$ 7.71-7.69 (d, 4H, $\left.\mathrm{H}_{\mathrm{e}}\right)$, 7.53-7.49 (m, 1H, Ha), 7.13-7.09 (m, 5H, $\mathrm{H}_{\mathrm{b}, \mathrm{d}}$ ), 7.05-7.03(m, 1H, Hc), 2.12 (s, 3H, $\left.\mathrm{H}_{\mathrm{CH}_{3}{ }^{\prime \prime}}\right), 1.29-1.23(\mathrm{~m}, 3 \mathrm{H}$, $\left.\mathrm{H}_{\mathrm{CH}_{3}}\right) .{ }^{13} \mathrm{C} \mathrm{NMR}\left(400 \mathrm{MHz}, \mathrm{CDCl}_{3}-d_{1}, \delta \mathrm{ppm}\right): 150.22\left(\mathrm{C}_{7}\right)$, 143.41 $\left(\mathrm{C}_{3}\right), 138.68\left(\mathrm{C}_{1}\right), 136.91\left(\mathrm{C}_{6}\right), 135.22\left(\mathrm{C}_{9}\right), 134.06\left(\mathrm{C}_{2}\right)$, $121.81\left(\mathrm{C}_{4}\right), 120.51\left(\mathrm{C}_{8}\right), 119.19\left(\mathrm{C}_{5}\right), 118.60\left(\mathrm{C}_{\mathrm{CN}}\right), 108.36\left(\mathrm{C}_{10}\right)$.<smiles>N#Cc1ccc(F)cc1</smiles><smiles>C=C(C)N(c1ccc(Br)cc1)c1ccc(C#N)cc1</smiles><smiles>C=C(C#N)C(C)OC(=O)c1ccc(N(c2ccc(C(=O)O)cc2)c2ccc(C(=O)O)cc2)cc1</smiles>

(Ia) -(Ic)

(Ma)-(Mc)<smiles>CCc1ccccc1C</smiles> 
4,4'-Dicarboxy-4"'-2,4-dimethyltriphenylamine (Mb): The synthesis of this compound was the same to that for Ma. The pale yellow crystals $3.40 \mathrm{~g}(94.18 \%$ yield $) ; \mathrm{m} . \mathrm{p}=276-$ $278{ }^{\circ} \mathrm{C}$. FTIR: $1683 \mathrm{~cm}^{-1}(\mathrm{C}=\mathrm{O}), 2710-3321 \mathrm{~cm}^{-1}(\mathrm{O}-\mathrm{H}) .{ }^{1} \mathrm{H}$ NMR (400 MHz, DMSO- $\left.d_{6}, \delta \mathrm{ppm}\right): 12.81\left(\mathrm{~s}, 2 \mathrm{H}, \mathrm{H}_{\mathrm{COOH}}\right)$, 7.98(d, 4H, $\left.\mathrm{H}_{\mathrm{e}}\right), 7.84-7.80\left(\mathrm{~m}, 1 \mathrm{H}, \mathrm{H}_{\mathrm{a}}\right), 7.14-7.12\left(\mathrm{~d}, 4 \mathrm{H}, \mathrm{H}_{\mathrm{d}}\right)$, 7.02-7.00(d, $\left.1 \mathrm{H}, \mathrm{H}_{\mathrm{b}}\right), 6.96-6.94\left(\mathrm{~d}, 1 \mathrm{H}, \mathrm{H}_{\mathrm{c}}\right), 2.06\left(\mathrm{~s}, 3 \mathrm{H}, \mathrm{H}_{\mathrm{CH} 3 "}\right)$, $1.20\left(\mathrm{~s}, 3 \mathrm{H}, \mathrm{H}_{\mathrm{CH}_{3}}\right) .{ }^{13} \mathrm{C}$ NMR $\left(400 \mathrm{MHz}, \mathrm{DMSO}-d_{6}, \delta \mathrm{ppm}\right)$ : $166.88\left(\mathrm{C}_{\mathrm{COOH}}\right), 149.86\left(\mathrm{C}_{7}\right), 143.47\left(\mathrm{C}_{3}\right), 132.00\left(\mathrm{C}_{9}\right)$, $131.11\left(\mathrm{C}_{1}\right), 129.00\left(\mathrm{C}_{2}\right), 128.02\left(\mathrm{C}_{6}\right), 122.04\left(\mathrm{C}_{4}\right), 126.76\left(\mathrm{C}_{10}\right)$ $120.29\left(\mathrm{C}_{5}\right), 118.98\left(\mathrm{C}_{8}\right), 33.15\left(\mathrm{C}_{\mathrm{CH}_{3}{ }}\right), 23.99\left(\mathrm{C}_{\mathrm{CH}_{3}{ }}\right)$.

4,4'-Dicyano-4"'-2-ethylaniline (Ic): The synthesis of this compound was the same to that for Ia. The product was filtered and recrystallized from ethanol to give pale yellowish solid 2.80 g (yield: $77.5 \%$ ), m.p. $=110-112^{\circ}$. FTIR: $2219 \mathrm{~cm}^{-1}(\mathrm{CN})$. ${ }^{1} \mathrm{H}$ NMR (400 MHz, $\left.\mathrm{CDCl}_{3}-d_{1}, \delta \mathrm{ppm}\right): 7.62-7.60\left(\mathrm{~d}, 4 \mathrm{H}, \mathrm{H}_{\mathrm{f}}\right.$ ), 7.43-7.31(m, 3H, $\left.\mathrm{H}_{\mathrm{a}, \mathrm{b}, \mathrm{c}}\right), 7.17-7.09$ (dd, 5H, $\left.\mathrm{H}_{\mathrm{e}, \mathrm{d}}\right), 2.39-2.32$ (q, 2H, $\mathrm{H}_{\mathrm{CH}_{2}}$ ), 0.99-0.96 (t, 3H, $\left.\mathrm{H}_{\mathrm{CH}_{3}}\right),{ }^{13} \mathrm{C} \mathrm{NMR}(400 \mathrm{MHz}$, $\left.\mathrm{CDCl}_{3}-d_{1}, \delta \mathrm{ppm}\right): 150.48\left(\mathrm{C}_{7}\right), 142.97\left(\mathrm{C}_{6}\right), 135.38-135.31$ $\left(\mathrm{C}_{1,5}\right), 134.04\left(\mathrm{C}_{9}\right), 131.34\left(\mathrm{C}_{2}\right), 130.59\left(\mathrm{C}_{3}\right), 129.06\left(\mathrm{C}_{4}\right)$, $122.33\left(\mathrm{C}_{8}\right) 120.54\left(\mathrm{C}_{\mathrm{CN}}\right), 105.51\left(\mathrm{C}_{10}\right), 24.46\left(\mathrm{C}_{\mathrm{CH}_{2}}\right), 13.86$ $\left(\mathrm{C}_{\mathrm{CH}_{3}}\right)$.

4,4'-Dicarboxy-4"'-2-ethyltriphenylamine (Mc): The synthesis of this compound was the same to that for Ma. The pale yellow crystals $3.40 \mathrm{~g}(94.2 \%$ yield $) ; \mathrm{m} . \mathrm{p} .=273-275^{\circ} \mathrm{C}$. FTIR: $1680 \mathrm{~cm}^{-1}(\mathrm{C}=\mathrm{O}), 2713-3332 \mathrm{~cm}^{-1}(\mathrm{O}-\mathrm{H}) .{ }^{1} \mathrm{H}$ NMR $(400$ $\left.\mathrm{MHz}, \mathrm{DMSO}-d_{6}, \delta \mathrm{ppm}\right): 12.76\left(\mathrm{~s}, 2 \mathrm{H}, \mathrm{H}_{\mathrm{COOH}}\right), 7.83-7.81(\mathrm{~d}$, $\left.4 \mathrm{H}, \mathrm{H}_{\mathrm{f}}\right), 7.42-7.31\left(\mathrm{~m}, 3 \mathrm{H}, \mathrm{H}_{\mathrm{a}, \mathrm{b}, \mathrm{c}}\right), 7.15-7.13\left(\mathrm{~d}, 5 \mathrm{H}, \mathrm{H}_{\mathrm{e}, \mathrm{d}}\right), 2.34-$ 2.28 (q, 2H, $\left.\mathrm{H}_{\mathrm{CH}_{2}}\right), 0.96-0.91\left(\mathrm{t}, 3 \mathrm{H}, \mathrm{H}_{\mathrm{CH}_{3}}\right) .{ }^{13} \mathrm{C} \mathrm{NMR}(400$ $\left.\mathrm{MHz}, \mathrm{DMSO}-d_{6}, \delta \mathrm{ppm}\right): 166.81\left(\mathrm{C}_{\mathrm{COOH}}\right), 150.86\left(\mathrm{C}_{7}\right)$, $143.25\left(\mathrm{C}_{6}\right), 131.20\left(\mathrm{C}_{1}\right) 131.03\left(\mathrm{C}_{5}\right), 132.18\left(\mathrm{C}_{9}\right), 128.50\left(\mathrm{C}_{2}\right)$, $128.34\left(\mathrm{C}_{3}\right), 128.17\left(\mathrm{C}_{4}\right), 126.33\left(\mathrm{C}_{10}\right), 122.16\left(\mathrm{C}_{8}\right) 23.15\left(\mathrm{C}_{\mathrm{CH}_{3}}\right)$, $13.23\left(\mathrm{C}_{\mathrm{CH}_{3}}\right)$.

\section{Polymer synthesis}

Synthesis of poly(amine-amide) (Pa1): The polymers (Pa1-Pc1, Pa2-Pc2) have synthesized via the routes as explained in the Scheme-II. The synthesis of poly(amine-amide) (Pa1) is used as an example to illustrate the general synthetic routes. The typical procedure is as follows. A mixture of $1.14 \mathrm{~g} \mathrm{(3}$ mmol) of the dicarboxylic acid monomer (Ma), $0.324 \mathrm{~g}$ (3 mmol) of $p$-phenylenediamine, $0.4 \mathrm{~g}$ of calcium chloride, 4.5 $\mathrm{mL}$ of triphenyl phosphite (TPP), $1.5 \mathrm{~mL}$ of pyridine and 4.5 $\mathrm{mL}$ of N-methyl-2-pyrrolidone (NMP) was heated with stirring at $105^{\circ} \mathrm{C}$ for $3 \mathrm{~h}$. The resulting viscous polymer solution was poured slowly into $300 \mathrm{~mL}$ of stirring methanol giving rise to a stringy, fiber-like precipitate that was collected by filtration, washed thoroughly with hot water and methanol and dried at $80^{\circ} \mathrm{C}$ for $24 \mathrm{~h}$. The inherent viscosity of the obtained poly(amine-amide) was $0.62 \mathrm{dL} / \mathrm{g}$, measured at a concentration of $0.5 \mathrm{~g} / \mathrm{dL}$ in dimethyl acetamide. The IR spectrum exhibited characteristic amide absorption bands at 3309.39 (N-H stretching) and $1644 \mathrm{~cm}^{-1}$ (amide carbonyl). ${ }^{1} \mathrm{H}$ NMR $(400 \mathrm{MHz}$, DMSO$\left.d_{6}, \delta \mathrm{ppm}\right): 10.25\left(\mathrm{~S}, 1 \mathrm{H}, \mathrm{H}_{\mathrm{NH}-\mathrm{CO}}\right), 8.05\left(\mathrm{~d}, 4 \mathrm{H}, \mathrm{H}_{\mathrm{d}}\right) 7.75(\mathrm{~s}, 4 \mathrm{H}$, $\left.\mathrm{H}_{\mathrm{e}}\right), 7.39\left(\mathrm{~d}, 2 \mathrm{H}, \mathrm{H}_{\mathrm{a}}\right), 7.20\left(\mathrm{~d}, 4 \mathrm{H}, \mathrm{H}_{\mathrm{c}}\right), 6.59$ (d, 2H, $\left.\mathrm{H}_{\mathrm{b}}\right), 3.19$ $\left(\mathrm{m}, 1 \mathrm{H}, \mathrm{H}_{\mathrm{CH}}\right) 2.07\left(\mathrm{~s}, 6 \mathrm{H}, \mathrm{H}_{\mathrm{CH}_{3}}\right) .{ }^{13} \mathrm{C} \mathrm{NMR}(400 \mathrm{MHz}$, DMSO$d_{6}, \delta$ ppm): 166.74 (NHCO), $150.44\left(\mathrm{C}_{5}\right), 145.82\left(\mathrm{C}_{4}\right), 143.22$ $\left(\mathrm{C}_{1}\right), 131.83\left(\mathrm{C}_{9}\right), 130.97\left(\mathrm{C}_{7}\right), 128.23\left(\mathrm{C}_{3}\right), 124.12\left(\mathrm{C}_{2}\right)$, $\left.126.12\left(\mathrm{C}_{8}\right), 121.76 \mathrm{C}_{6}\right), 120.02\left(\mathrm{C}_{10}\right), 32.91\left(\mathrm{C}_{\mathrm{CH}}\right), 23.81\left(\mathrm{C}_{\mathrm{CH}_{3}}\right)$. The other poly(amine-amide)s were prepared by analogous procedure.

Synthesis of poly(amine-amide) (Pb1): The polymer Pb1 was synthesized by the same method as described in synthesis of Pa1. The obtained poly(amine-amide) was 0.66 $\mathrm{dL} / \mathrm{g}$, measured at a concentration of $0.5 \mathrm{~g} / \mathrm{dL}$ in DMAc. The IR spectrum $3304 \mathrm{~cm}^{-1}$ (N-H stretching) and $1644 \mathrm{~cm}^{-1}$ (amide carbonyl). ${ }^{1} \mathrm{H}$ NMR (400 MHz, DMSO- $d_{6}, \delta$ ppm): 10.26 (s, $\left.1 \mathrm{H}, \mathrm{H}_{\mathrm{NHCO}}\right)$, 8.05-7.90 (d, 5H, $\left.\mathrm{H}_{\mathrm{e}}\right), 7.74\left(\mathrm{~s}, 4 \mathrm{H}, \mathrm{H}_{\mathrm{f}}\right), 7.20(\mathrm{~s}$, $\left.4 \mathrm{H}, \mathrm{H}_{\mathrm{a}, \mathrm{b}, \mathrm{d}}\right), 6.74\left(\mathrm{~s}, 1 \mathrm{H}, \mathrm{H}_{\mathrm{b}}\right), 6.74-6.56\left(\mathrm{~s}, 1 \mathrm{H}, \mathrm{H}_{\mathrm{c}}\right), 1.97(\mathrm{~s}, 3 \mathrm{H}$, $\left.\mathrm{H}_{\left.\mathrm{CH}_{3}{ }{ }\right)}\right) 1.21\left(\mathrm{~s}, 3 \mathrm{H}, \mathrm{H}_{\mathrm{CH}_{3}}{ }\right) .{ }^{13} \mathrm{C} \mathrm{NMR}\left(400 \mathrm{MHz}, \mathrm{DMSO}-d_{6}, \delta\right.$ ppm): $166.89\left(\mathrm{C}_{\mathrm{CO}-\mathrm{NH}}\right), 149.97\left(\mathrm{C}_{7}\right), 142.89\left(\mathrm{C}_{3}\right), 131.91\left(\mathrm{C}_{9}\right)$, 131.21 $\left(\mathrm{C}_{1}\right), 129.04\left(\mathrm{C}_{2}\right), 128.34\left(\mathrm{C}_{6}\right), 126.45\left(\mathrm{C}_{10}\right), 125.22\left(\mathrm{C}_{11}\right)$, 123.17( $\left(\mathrm{C}_{4}\right), 120.17\left(\mathrm{C}_{5}\right), 118.12\left(\mathrm{C}_{8}\right), 117.23\left(\mathrm{C}_{12}\right), 33.45\left(\mathrm{C}_{\mathrm{CH}_{3}{ }^{\prime \prime}}\right)$.

Synthesis of poly(amine-amide) (Pc1): The polymer Pb1 was synthesized by the same method as described in synthesis of Pa1. The obtained poly(amine-amide) was $0.63 \mathrm{dL} / \mathrm{g}$,

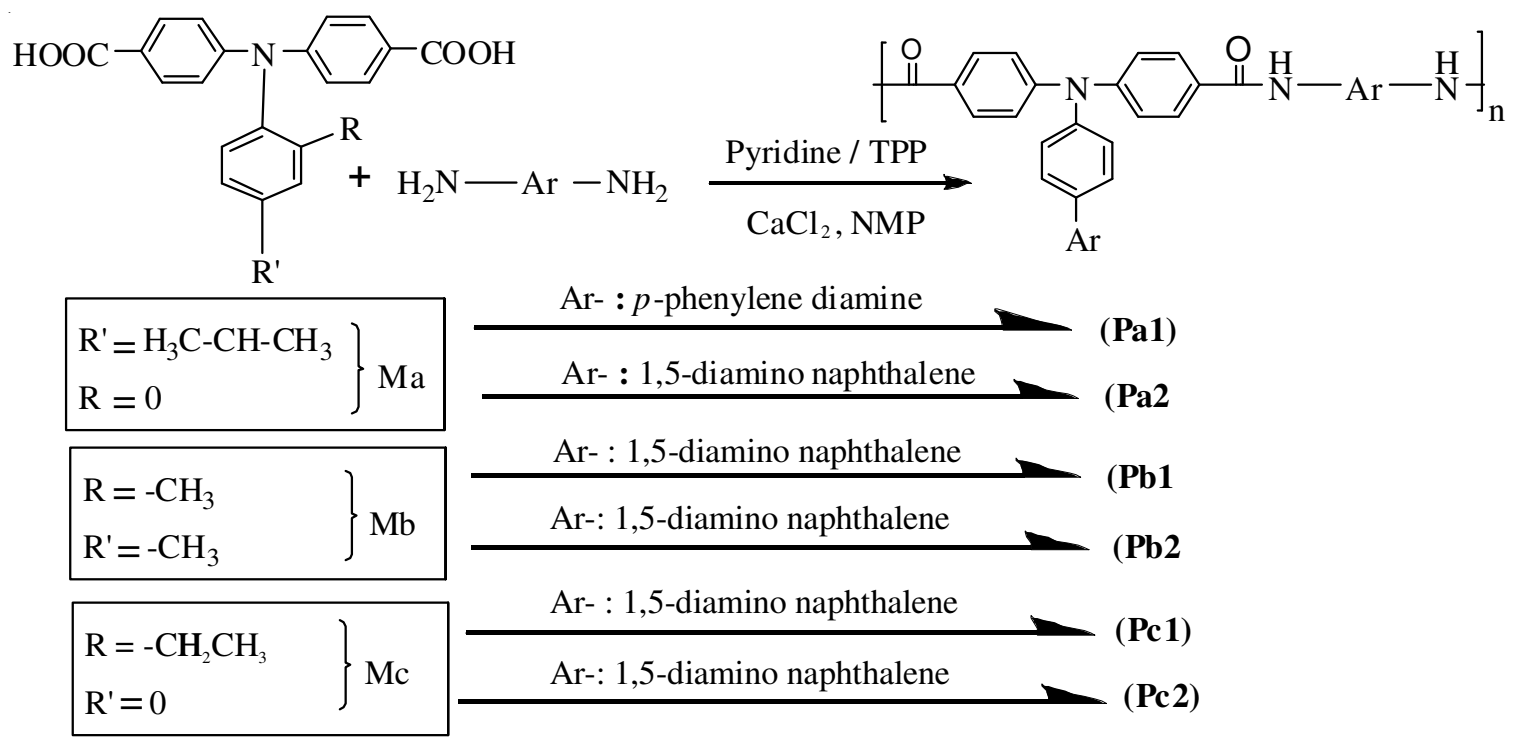

Scheme-II: Synthetic routes of the polymers 
measured at a concentration of $0.5 \mathrm{~g} / \mathrm{dL}$ in dimethyl acetamide. The IR spectrum $3314 \mathrm{~cm}^{-1}$ (N-H stretching) and $1644 \mathrm{~cm}^{-1}$ (amide carbonyl). ${ }^{1} \mathrm{H}$ NMR $\left(400 \mathrm{MHz}, \mathrm{DMSO}-d_{6}, \delta\right.$ ppm):10.23(S, 1H, $\left.\mathrm{H}_{\mathrm{NHCO}}\right)$, 7.98-7.83 (S, 1H, $\left.\mathrm{H}_{\mathrm{f}, \mathrm{a}}\right), 7.40-7.34$ $\left(\mathrm{S}, 1 \mathrm{H}, \mathrm{H}_{\mathrm{g}, \mathrm{c}}\right), 7.13-6.73\left(\mathrm{~S}, 1 \mathrm{H}, \mathrm{H}_{\mathrm{b}, \mathrm{e}, \mathrm{d}}\right), 2.06\left(\mathrm{~S}, 1 \mathrm{H}, \mathrm{H}_{\mathrm{CH} 2}\right), 0.93(\mathrm{~S}$, $\left.1 \mathrm{H}, \mathrm{H}_{\mathrm{CH}_{3}}\right){ }^{13} \mathrm{C}$ NMR (400 MHz, DMSO- $\left.d_{6}, \delta \mathrm{ppm}\right): 166.82$ $\left(\mathrm{C}_{\mathrm{CO}-\mathrm{NH}}\right), 150.12\left(\mathrm{C}_{7}\right), 142.89\left(\mathrm{C}_{6}\right), 131.20\left(\mathrm{C}_{1}\right), 131.03\left(\mathrm{C}_{5}\right)$, 131.91( $\left(C_{9}\right), 129.17\left(C_{2}\right), 128.89\left(C_{3}\right), 127.16\left(C_{4}\right), 126.12\left(C_{10}\right)$, $125.32\left(\mathrm{C}_{11}\right), 122.23\left(\mathrm{C}_{8}\right), 121.18\left(\mathrm{C}_{12}\right), 26.45\left(\mathrm{C}_{\mathrm{CH} 2}\right)$, $13.43\left(\mathrm{C}_{\mathrm{CH}_{3}}\right)$.

Synthesis of poly(amine-amide) (Pa2): This polymer Pa2 was synthesized by the same method as described in synthesis of Pa1 but used 1,5-diamine naphthalene instead of $p$-phenylene diamine.The inherent viscosity of the obtained poly(amine-amide) was $0.75 \mathrm{dL} / \mathrm{g}$, measured at a concentration of $0.5 \mathrm{~g} / \mathrm{dL}$ in DMAc. The IR spectrum exhibited characteristic amide absorption bands at $3364 \mathrm{~cm}^{-1}$ (N-H stretching) and $1647 \mathrm{~cm}^{-1}$ (amide carbonyl). ${ }^{1} \mathrm{H}$ NMR (400 MHz, DMSO- $d_{6}$, $\delta \mathrm{ppm}): 10.42\left(\mathrm{~s}, 1 \mathrm{H}, \mathrm{H}_{\mathrm{NH}-\mathrm{CO}}\right), 7.99-7.97\left(\mathrm{~d}, 2 \mathrm{H}, \mathrm{H}_{\mathrm{g}}\right), 7.84-$ 7.82(d, $\left.4 \mathrm{H}, \mathrm{H}_{\mathrm{d}}\right), 7.50\left(\mathrm{~s}, 2 \mathrm{H}, \mathrm{H}_{\mathrm{f}}\right), 7.37-7.35\left(\mathrm{~d}, 2 \mathrm{H}, \mathrm{H}_{\mathrm{a}}\right), 7.29-$ 7.27(d, $\left.2 \mathrm{H}, \mathrm{H}_{\mathrm{e}}\right), 7.15-7.13\left(\mathrm{~d}, 4 \mathrm{H}, \mathrm{H}_{\mathrm{c}}\right), 7.03-7.01\left(\mathrm{~d}, 2 \mathrm{H}, \mathrm{H}_{\mathrm{b}}\right)$, $1.92-1.84\left(\mathrm{~m}, 1 \mathrm{H}, \mathrm{H}_{\mathrm{CH}}\right), 1.21-1.19\left(\mathrm{~d}, 6 \mathrm{H}, \mathrm{H}_{\mathrm{CH}_{3}}\right) .{ }^{13} \mathrm{C} \mathrm{NMR}(400$ MHz, DMSO- $\left.d_{6}, \delta \mathrm{ppm}\right): 166.56\left(\mathrm{C}_{\mathrm{CONH}}\right), 150.47\left(\mathrm{C}_{5}\right), 145.68$ $\left(\mathrm{C}_{4}\right), 143.46\left(\mathrm{C}_{1}\right), 131.76\left(\mathrm{C}_{9}\right), 130.36\left(\mathrm{C}_{7}\right), 128.33\left(\mathrm{C}_{3}\right)$, $127.40\left(\mathrm{C}_{2}\right), 126.11\left(\mathrm{C}_{8}\right), 125.98\left(\mathrm{C}_{13}\right), 125.68\left(\mathrm{C}_{11}\right), 121.86\left(\mathrm{C}_{6}\right)$, $110.77\left(\mathrm{C}_{12}\right), 104.24\left(\mathrm{C}_{10}\right), 32.82\left(\mathrm{C}_{\mathrm{CH}}\right), 23.87\left(\mathrm{C}_{\mathrm{CH}_{3}}\right)$.

Synthesis of poly(amine-amide) (Pb2): This polymer was synthesized by the same method ass describd in synthesis of Pa1 but used 1,5-diamine naphthalene instead of $p$-phenylene diamine. The inherent viscosity of the obtained poly(amineamide) was $0.67 \mathrm{dL} / \mathrm{g}$, measured at a concentration of 0.5 $\mathrm{g} / \mathrm{dL}$ in DMAc. The IR spectrum exhibited characteristic amide absorption bands at $3364 \mathrm{~cm}^{-1}$ (N-H stretching) and $1648 \mathrm{~cm}^{-1}$ (amide carbonyl). ${ }^{1} \mathrm{H}$ NMR $\left(400 \mathrm{MHz}, \mathrm{DMSO}-d_{6}, \delta \mathrm{ppm}\right)$ : $10.46\left(\mathrm{~s}, 1 \mathrm{H}, \mathrm{H}_{\mathrm{NH}-\mathrm{CO}}\right), 8.14$ (s, 6H, $\left.\mathrm{H}_{\mathrm{e}, \mathrm{h}}\right), 7.94-7.88\left(\mathrm{~d}, 2 \mathrm{H}, \mathrm{H}_{\mathrm{g}}\right)$, 7.55-7.24(d, $\left.2 \mathrm{H}, \mathrm{H}_{\mathrm{f}}\right), 7.20-7.06\left(\mathrm{~d}, 6 \mathrm{H}, \mathrm{H}_{\mathrm{a}, \mathrm{b}, \mathrm{d}}\right), 6.68-6.64(\mathrm{~d}, 2 \mathrm{H}$, $\left.\mathrm{H}_{\mathrm{c}}\right), 1.98-1.76\left(\mathrm{~s}, 3 \mathrm{H}, \mathrm{H}_{\mathrm{CH}_{3}{ }^{\prime}}\right), 1.23-0.92\left(\mathrm{~s}, 3 \mathrm{H}, \mathrm{H}_{\mathrm{CH}_{3}}\right)$ ). ${ }^{13} \mathrm{C} \mathrm{NMR}$ (400 MHz, DMSO- $\left.d_{6}, \delta \mathrm{ppm}\right): 166.86\left(\mathrm{C}_{\mathrm{CONH}}\right), 150.54\left(\mathrm{C}_{7}\right)$, $143.73\left(\mathrm{C}_{3}\right), 132.71\left(\mathrm{C}_{11}\right), 131.75\left(\mathrm{C}_{9}\right), 131.12\left(\mathrm{C}_{1}\right), 130.76\left(\mathrm{C}_{2}\right)$, $128.58\left(\mathrm{C}_{6}\right), \quad 126.67\left(\mathrm{C}_{10}\right), \quad 125.87\left(\mathrm{C}_{15}\right), \quad 124.77\left(\mathrm{C}_{13}\right)$, $122.57\left(\mathrm{C}_{4}\right), 121.82\left(\mathrm{C}_{5}\right) .118 .82\left(\mathrm{C}_{8}\right), 110.23\left(\mathrm{C}_{14}\right), 107.63\left(\mathrm{C}_{12}\right)$, 33.07( $\left(\mathrm{C}_{\mathrm{CH}_{3}}{ }^{\prime \prime}\right), 23.65\left(\mathrm{C}_{\mathrm{CH}_{3}}\right)$.

Synthesis of poly(amine-amide) (Pc2): This polymer Pc2 was synthesized by the same method as described in synthesis of Pa1 but used 1,5-diamine naphthalene instead of $p$-phenylene diamine.The inherent viscosity of the obtained poly(amine-amide) was $0.81 \mathrm{dL} / \mathrm{g}$, measured at a concentration of $0.5 \mathrm{~g} / \mathrm{dL}$ in DMAc. The IR spectrum exhibited characteristic amide absorption bands at $3357 \mathrm{~cm}^{-1}$ (N-H stretching) and $1648 \mathrm{~cm}^{-1}$ (amide carbonyl). ${ }^{1} \mathrm{H}$ NMR $\left(400 \mathrm{MHz}, \mathrm{DMSO}-d_{6}\right.$, $\delta \mathrm{ppm}): 10.43\left(\mathrm{~s}, 1 \mathrm{H}, \mathrm{H}_{\mathrm{NHCO}}\right), 8.12-8.08\left(\mathrm{~s}, 7 \mathrm{H}, \mathrm{H}_{\mathrm{f}, \mathrm{a}, \mathrm{i}}\right), 7.88-7.86$ $\left(\mathrm{d}, 2 \mathrm{H}, \mathrm{H}_{\mathrm{h}}\right), 7.64-7.50\left(\mathrm{~d}, 2 \mathrm{H}, \mathrm{H}_{\mathrm{g}, \mathrm{c}}\right), 7.19-7.00\left(\mathrm{~d}, 5 \mathrm{H}, \mathrm{H}_{\mathrm{e}, \mathrm{d}}\right)$,

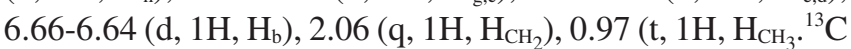
NMR (400 MHz, DMSO- $d_{6}, \delta$ ppm): 165.92 ( $\left.\mathrm{C}_{\mathrm{CONH}}\right), 150.24$ $\left(\mathrm{C}_{7}\right), 142.56\left(\mathrm{C}_{6}\right), 132.05\left(\mathrm{C}_{5}\right), 131.85\left(\mathrm{C}_{1}\right), 131.24\left(\mathrm{C}_{11}\right)$, $130.86\left(\mathrm{C}_{9}\right), 131.12\left(\mathrm{C}_{2}\right), 130.76\left(\mathrm{C}_{3}\right), 128.58\left(\mathrm{C}_{4}\right), 126.56\left(\mathrm{C}_{10}\right)$, $124.06\left(\mathrm{C}_{15}\right), 121.77\left(\mathrm{C}_{13}\right), \quad 118.57\left(\mathrm{C}_{8}\right), 110.32\left(\mathrm{C}_{14}\right)$. $106.81\left(\mathrm{C}_{12}\right), 26.43\left(\mathrm{C}_{\mathrm{CH}_{2}}\right), 13.63\left(\mathrm{C}_{\mathrm{CH}_{3}}\right)$.

\section{RESULTS AND DISCUSSION}

Monomer synthesis: The new aromatic dicarboxylic acid having 4-isopropyl substituted triphenylamine unit, 4,4'dicarboxy-4"-isopropyltriphenylamine (Ma), was synthesized by the amination reaction of 4-isopropylaniline with 4-fluorobenzonitrile, followed by the alkaline hydrolysis of the intermediate dicyano compound (Ia). According to the synthetic routes outlined in Scheme-I. FT IR, ${ }^{1} \mathrm{H}$ and ${ }^{13} \mathrm{C}$ NMR spectroscopic techniques were used to identify the chemical structures of the intermediate dicyano compounds (Ia) and the dicarboxylic acid monomer (Ma).The FTIR spectra of compound Ia gave a cyano group characteristic band at 2221 $\mathrm{cm}^{-1}$ ( $\mathrm{C} \equiv \mathrm{N}$ stretching). After hydrolysis, the cyano group absorption beak disappeared and the carboxylic acid group showed a typical carbonyl absorption band at $1678 \mathrm{~cm}^{-1}(\mathrm{C}=\mathrm{O}$ stretching) together with the appearance of broad bands around 3400-2700 $\mathrm{cm}^{-1}$ (O-H stretching) (Fig. 1).

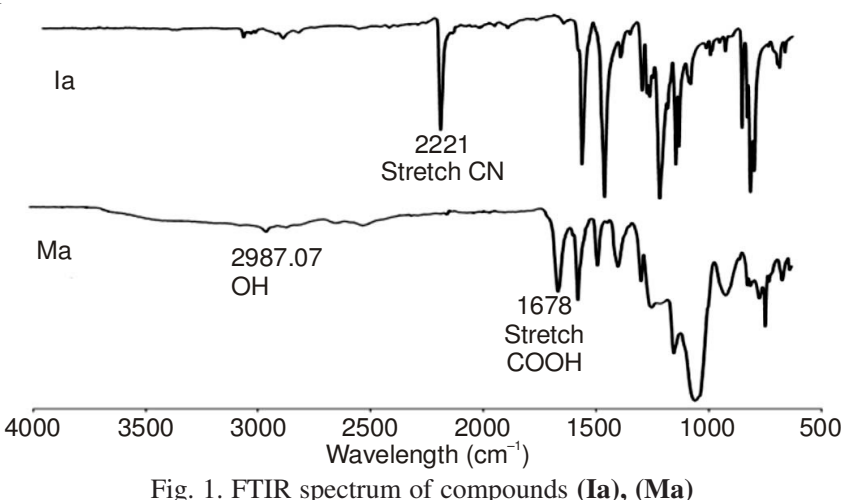

Structures of compounds Ia and Ma were also confirmed by high-resolution NMR spectra (Figs. 2 and 3). In ${ }^{1} \mathrm{H}$ NMR spectrum (Fig. 2), a two big doublet peaks at 7.42 (d, $4 \mathrm{H}, \mathrm{H}_{\mathrm{d}}$ ), $7.03\left(\mathrm{~d}, 4 \mathrm{H}, \mathrm{H}_{\mathrm{c}}\right)$, due to four phenylene protons $(\mathrm{d})$ and $(\mathrm{c})$, respectively. Doublet peak at $7.15(\mathrm{~d}, 2 \mathrm{H}, \mathrm{Ha})$ due to two protons of (a). Doublet peak at $6.97\left(\mathrm{~d}, 2 \mathrm{H}, \mathrm{H}_{\mathrm{b}}\right)$ for two protons of (b). Multiplet peak of proton (-CH-). Doublet peak of six protons for $\left(2 \mathrm{CH}_{3}\right)$. Fig. 3 exhibits very broad singlet peak due to proton of carboxylic group which is hardly observed because replacement hydrogen atoms of $(\mathrm{COOH})$ by deuterium of DMSO solvent and formation of $\mathrm{H}$-bonding. The ${ }^{13} \mathrm{C} \mathrm{NMR}$ spectra (Figs. 4 and 5) confirmed that the chemical shifting of $\mathrm{C}$-atoms and that the cyano groups were completely converted into the carboxylic acid groups by the disappearance of the resonance peak for the cyano carbon at $105.42 \mathrm{ppm}$ and by the appearance of the carboxylic peak at $166.8 \mathrm{ppm}$.

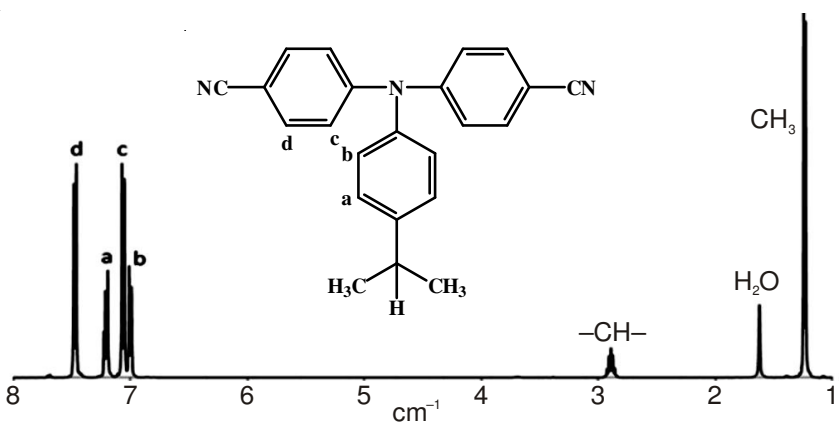

Fig. 2. ${ }^{1} \mathrm{H}$ NMR spectrum of compound (Ia) in $\mathrm{CDCl}_{3}-\mathrm{D} 1$ 


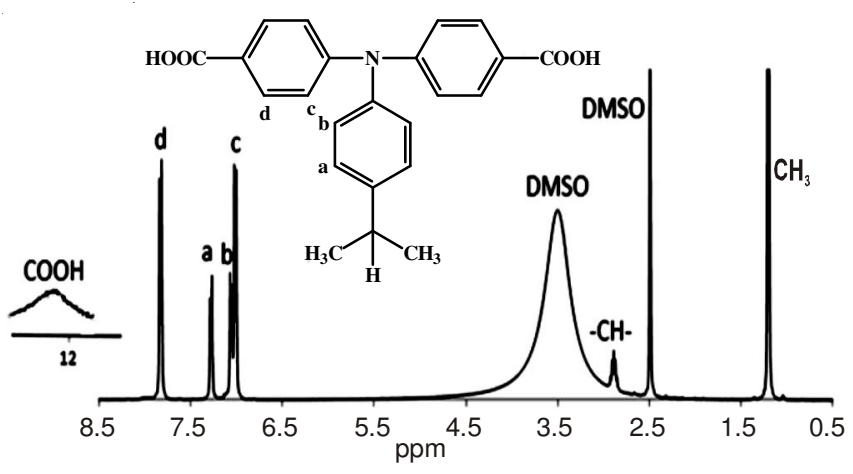

Fig. 3. ${ }^{1} \mathrm{H}$ NMR spectrum of compound (Ma) in $\mathrm{CDCl}_{3}-\mathrm{D} 1$

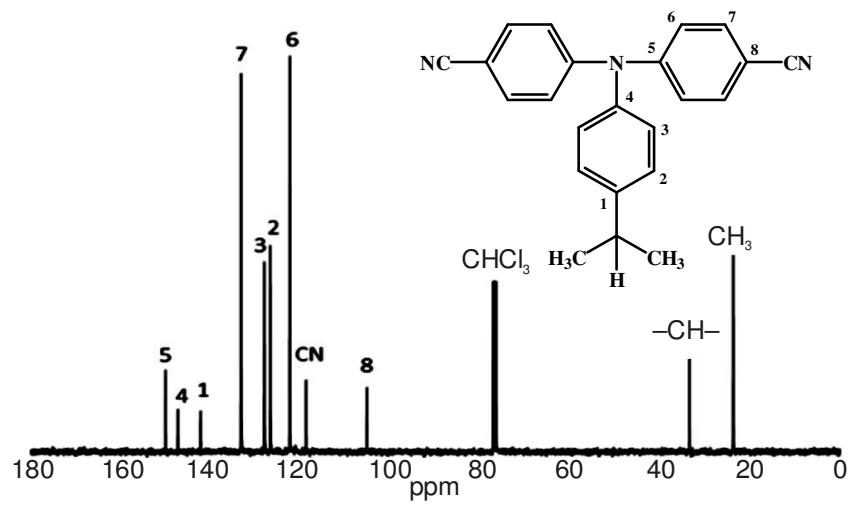

Fig. $4 .{ }^{13} \mathrm{C}$ NMR spectrum of compound (Ia) in $\mathrm{CDCl}_{3}-\mathrm{D} 1$

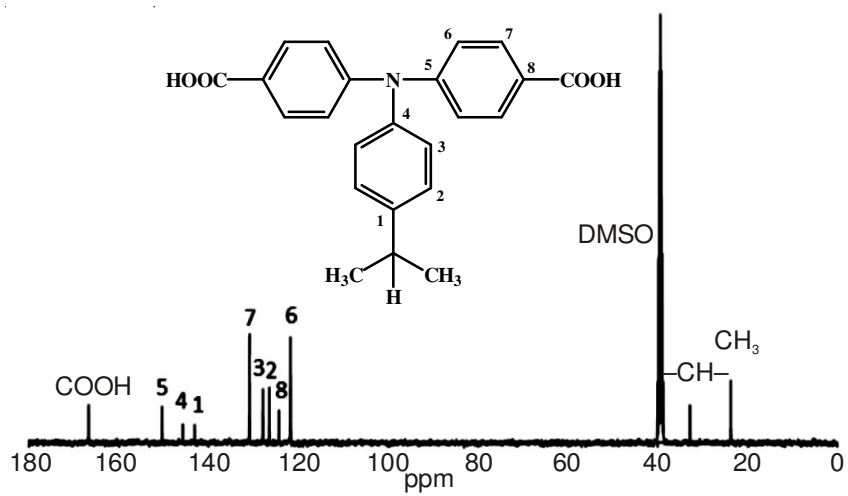

Fig. $5 .{ }^{13} \mathrm{C}$ NMR spectrum of compound (Ma) in DMSO- $d_{6}$

\begin{tabular}{ccc} 
& $\begin{array}{c}\text { TABLE-1 } \\
\text { POLYMER CODES }\end{array}$ \\
\hline & Polymer diamine & Monomer code \\
\hline pa1 & 1 & Ma \\
pb1 & 1 & Mb \\
pc1 & 1 & Mc \\
pa2 & 2 & Ma \\
pb2 & 2 & Mb \\
pc2 & 2 & Mc \\
\hline
\end{tabular}

Other important evidence of this change is the shifting of the carbon resonance signals of $\mathrm{C}_{8}$ adjacent to the cyano or carboxyl group. The $\mathrm{C}_{8}$ of dinitrile (Ia) resonated at a higher field $(105.4 \mathrm{ppm})$ than the other aromatic carbons because of the anisotropic shielding by the $\pi$-electrons of $\mathrm{Ca} \equiv \mathrm{N}$. After hydrolysis, the resonance peak of $\mathrm{C}_{8}$ shifted to a lower field (126.56 ppm) because of the lack of an anisotropic field.

Polymer synthesis: A series of new aromatic poly(amineamide)s with aryltriphenylamine (ATPA) units were prepared by the direct polycondensation reactions of the dicarboxylic acid monomer (Ma) with various aromatic diamines, $p$-phenylenediamine, 1,5-diamino naphthalene using triphenylphosphite (TPP) and pyridine as condensing agents (Scheme-II). All the polymerizations proceeded homogeneously throughout the reaction and afforded clear, highly viscous polymer solutions. These polymers precipitated in a tough, fiber-like form when the resulting polymer solutions were slowly poured with stirring into methanol. These poly(amine-amide)s were obtained in almost quantitative yields, with $\eta_{\text {Cinh }}$ values in the range of 0.62-0.81 dL/g (Table-2). All the polymers can be solutioncast into flexible and tough films. Structural features of these poly(amine-amide)s were verified by FTIR and NMR spectroscopy. The characteristic absorption bands observed around $3309.39\left(\mathrm{~N}-\mathrm{H}\right.$ stretching) and $1644 \mathrm{~cm}^{-1}(\mathrm{C}=\mathrm{O}$ stretching of amide group). Fig. 6 illustrates a typical FTIR spectrum of the representative poly(amine-amide) (pa1). Typical set of ${ }^{1} \mathrm{H}$ and ${ }^{13} \mathrm{C}$ NMR spectra of polyamide in DMSO- $d_{6}$. Assignments of each proton and the spectra agree well with the proposed molecular structure of pa1.
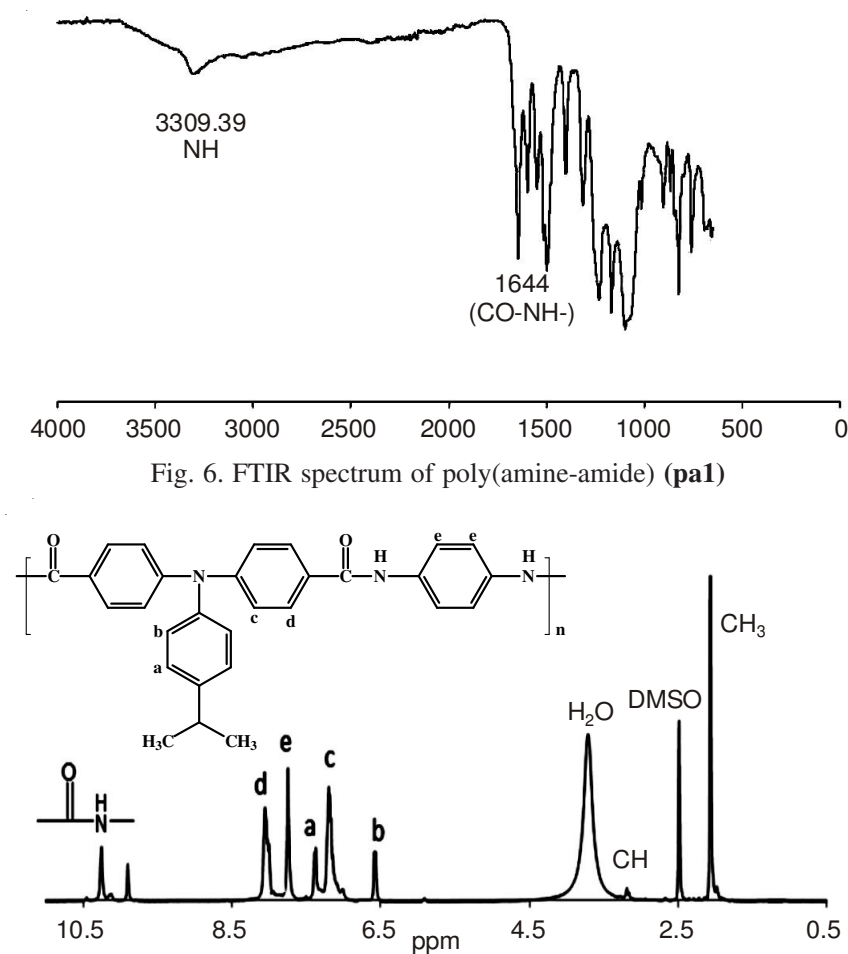

Fig. 7. ${ }^{1} \mathrm{H}$ NMR of compound poly(amine-amide) (pa1)

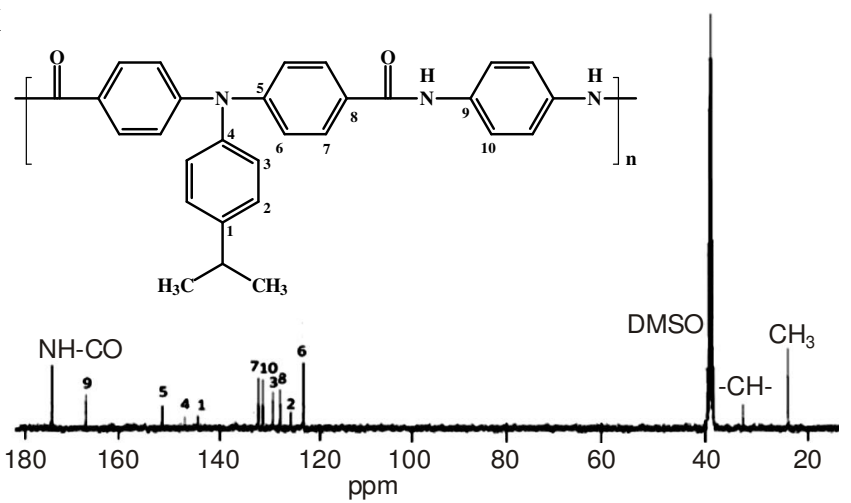

Fig. 8. ${ }^{13} \mathrm{C}$ NMR of poly(amine-amide) (Pa1) in DMSO- $d_{6}$ 
TABLE-2

SOLUBILITY OF AROMATIC POLYAMIDES

\begin{tabular}{|c|c|c|c|c|c|c|c|c|}
\hline \multirow{2}{*}{$\begin{array}{l}\text { Polymer } \\
\text { code }\end{array}$} & \multirow{2}{*}{$\operatorname{inh}^{\mathrm{b}}(\mathrm{dl} / \mathrm{g})$} & \multicolumn{7}{|c|}{ Solvents $^{\mathrm{a}}$} \\
\hline & & NMP & DMAc & DMF & DMSO & $m$-Cresol & $\mathrm{THF}$ & $\mathrm{CHCl}_{3}$ \\
\hline pal1 & 0.62 & ++ & ++ & ++ & ++ & - & - & - \\
\hline pb1 & 0.66 & ++ & ++ & ++ & ++ & - & - & - \\
\hline pcl1 & 0.63 & ++ & ++ & ++ & ++ & - & - & - \\
\hline pa2 & 0.75 & ++ & ++ & ++ & ++ & + & - & - \\
\hline pb2 & 0.67 & ++ & ++ & ++ & ++ & + & - & - \\
\hline pc2 & 0.81 & ++ & ++ & ++ & ++ & + & - & - \\
\hline
\end{tabular}

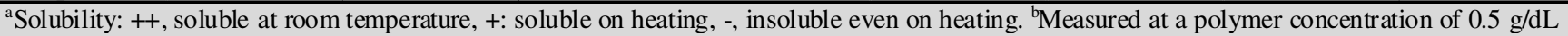
in DMAc at $30^{\circ} \mathrm{C}$.

Also, the monomer Ma reacts with 1,5-diamino naphthalene to form polyamide with more solubility in the polar solvent. This polymer characterized by FTIR, ${ }^{1} \mathrm{H}$ and ${ }^{13} \mathrm{C}$ NMR as shown in Figs. 9-11.

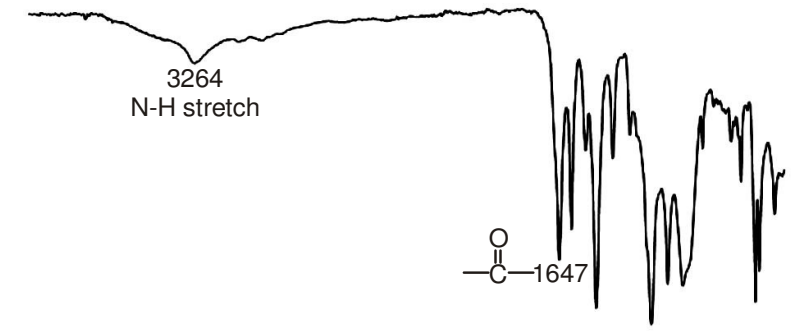

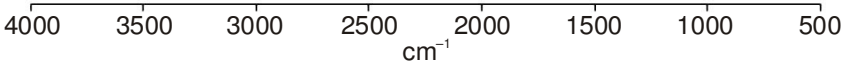

Fig. 9. FTIR spectrum of poly(amine-amide) (pa2)

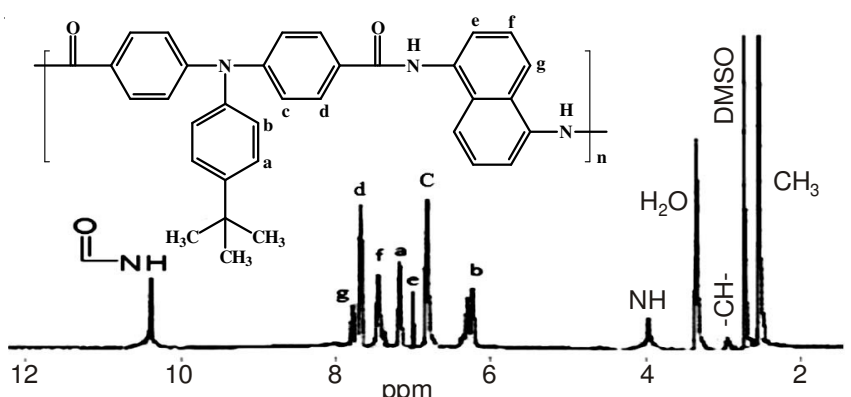

Fig. 10. ${ }^{1} \mathrm{H}$ NMR of compound poly(amine-amide) (pa2)

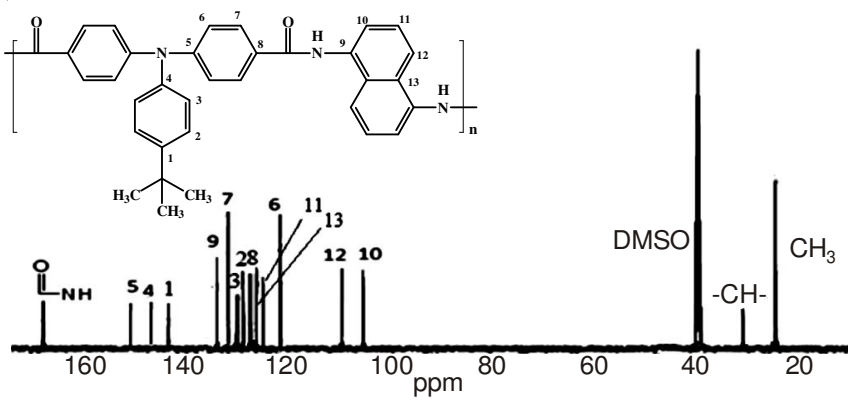

Fig. 11. ${ }^{13} \mathrm{C}$ NMR of poly(amine-amide) (Pa2)

\section{Polymer properties}

Solubility: The qualitative solubility properties of poly(amine-amide)s in several organic solvents at $10 \%(\mathrm{w} / \mathrm{v})$ are also summarized in Table-2. All the polymers exhibited excellent solubility in polar organic solvents such as NMP and DMAc. The enhanced solubility can be attributed to the presence of introduced triphenylamine unit. Polymers (pa2), (pb2), (pc2) have more solubility than others because the more bulky pendent naphthalene group chromophores in the repeating unit leading to increase in free volume in the polymer chains to allow more solvent to come in. Further more, polymers (pb2)-(pc2) are more flexibility. The excellent solubility makes these polymers convenient to process into articles by spin-coating.

Thermal properties: DSC thermograms of all the poly(amine-amide)sare as shown in Fig. 12. The thermal behaviour data of these polymers are summarized in Table-3. They showed high glass transition temperatures $\mathrm{T}_{\mathrm{g}}$ in the range of (289.60-248.32) ${ }^{\circ} \mathrm{C}$. The $\mathrm{T}_{\mathrm{g}}$ values of these poly(amineamide)s generally decreased with decreasing stiffness of the diamine component. So polymers with phenylene diamine exhibited high $\mathrm{T}_{\mathrm{g}}$ value due to more rigidity than of polymers with 1,5-diamine. The lowest $\mathrm{T}_{\mathrm{g}}$ value of poly(amine-amide)s can be explained in terms of the flexibility and low rotation barrier of its diamine moiety. Poly(amine-amide) (pb1) exhibited the highest $\mathrm{T}_{\mathrm{g}}$ value $\left(289.60{ }^{\circ} \mathrm{C}\right)$ in this series polymers because of the presence of rigid phenylene diamine and methyl groups segment in the main chain.

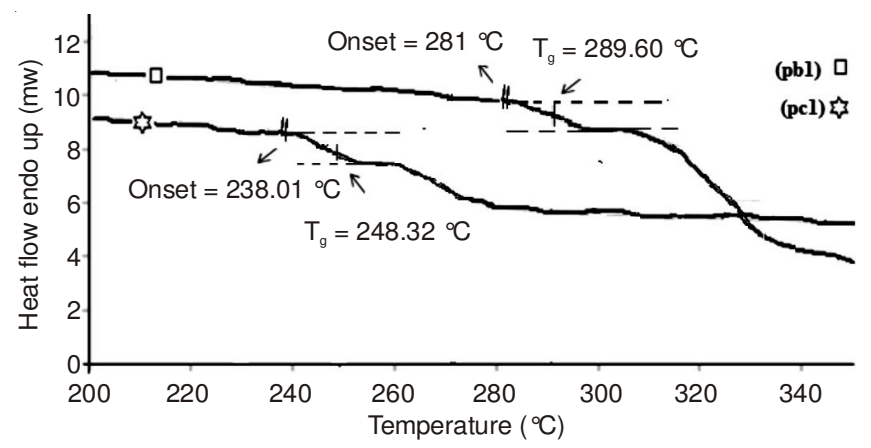

Fig. 12. DSC of poly(amine-amide)s (pa1), (pc1)

Fig. 13 shows the TGA curves of poly(amine-amide) (pa1). The decomposition temperatures $\left(\mathrm{T}_{\mathrm{d}}\right)$ at 5 and $10 \%$ weight losses in nitrogen atmosphere are given in Table-3. All the poly(amine-amide)s exhibited good thermal stability with insignificant weight loss up to $300{ }^{\circ} \mathrm{C}$ in nitrogenand the $5 \%$ weight-loss temperatures in nitrogen were recorded in the range of $\left(410-460{ }^{\circ} \mathrm{C}\right)$ and $\left(451-522^{\circ} \mathrm{C}\right)$ for $10 \%$ weight. Due to their high aromatic content, these polymers revealed high char yields in nitrogen, more than $60 \%$ at $800{ }^{\circ} \mathrm{C}$.

Spectral properties: The optical properties of poly(amine-amide)s were investigated by UV-visible and photo- 


\begin{tabular}{cccccccc}
\hline & \multicolumn{7}{c}{ TABLE-3 } \\
\hline Polymer code $\left({ }^{\circ} \mathrm{C}\right)$ & Onset $^{\mathrm{a}}\left({ }^{\circ} \mathrm{C}\right)$ & $\mathrm{T}_{\mathrm{b}}^{\mathrm{b}}\left({ }^{\circ} \mathrm{C}\right)$ & Onset $^{\mathrm{c}}\left({ }^{\circ} \mathrm{C}\right)$ & $\mathrm{T}^{\mathrm{d}}$ 5 \% $\left({ }^{\circ} \mathrm{C}\right)$ & $\mathrm{T}^{\mathrm{d}}$ 10\% $\left({ }^{\circ} \mathrm{C}\right)$ & Peak $(\%)$ & Char. $^{\mathrm{e}}$ \\
\hline$(\mathrm{pb} 1)$ & 265.45 & 283.32 & 383.76 & 460 & 522 & 444.04 & 63 \\
$(\mathrm{pc} 1)$ & 281.00 & 289.60 & 364.84 & 410 & 480 & 443.72 & 65 \\
$(\mathrm{p} 1)$ & 230.01 & 248.32 & 367.37 & 400 & 475 & 437.20 & 67 \\
$(\mathrm{pa} 2)$ & 243.32 & 260.00 & 357.04 & 400 & 475 & 421.22 & 61 \\
$(\mathrm{pb} 2)$ & 246.92 & 265.45 & 361.43 & 430 & 472 & 411.04 & 65 \\
$(\mathrm{pc} 2)$ & 238.30 & 248.32 & 370.21 & 420 & 451 & 405.23 & 65 \\
\hline
\end{tabular}

a: onset temp,recorded by DSC. b: The midpoint temperature of baseline shift on the subsequent DSC trace ( from $30-350{ }^{\circ} \mathrm{C}$ at heating rate 20 ${ }^{\circ} \mathrm{C} / \mathrm{min}$ ) was defined as $\mathrm{T}_{\mathrm{g}}$. C: onset temp. Recorded by TGA. $\mathrm{T}_{\mathrm{d}}$ : Decomposition temperature at which a $5 \%$ or $10 \%$ weight loss was recorded by TGA at a heating rate of $20^{\circ} \mathrm{C} / \mathrm{min}$. Peak: The midpoint temperature of baseline shift on the subsequent TGA trace (from $50-800{ }^{\circ} \mathrm{C}$ at heating rate $\left.20^{\circ} \mathrm{C} / \mathrm{min}\right)$ was defined as peak $\left({ }^{\circ} \mathrm{C}\right)$. e: Residual weight percentages at $800{ }^{\circ} \mathrm{C}$ under nitrogen flow.

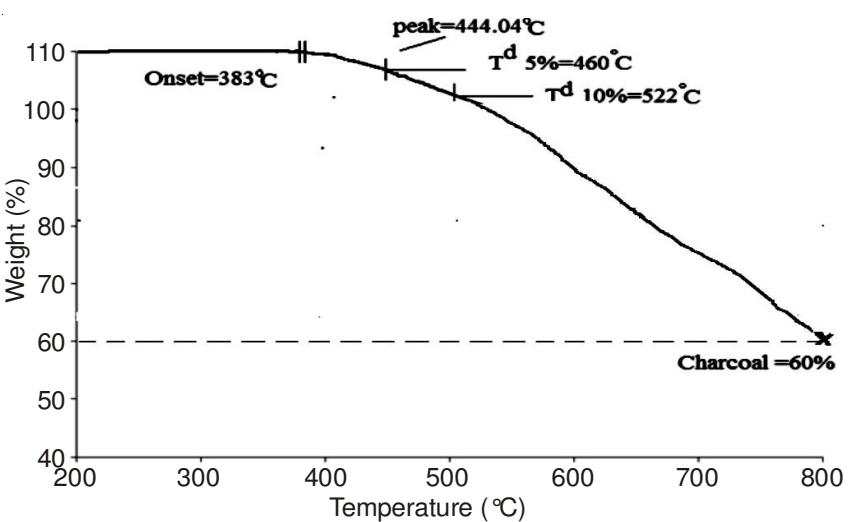

Fig. 13. TGA of Poly(amin-amide) (pa1)

luminescence spectroscopy. The results are summarized in Table-4. The dilute solutions of these poly(amine-amide)s in NMP exhibited strong UV-VIS absorption bands at (318-355) $\mathrm{nm}$, assignable to the $\left(\mathrm{n}-\pi^{*}\right)$ transition resulting from the conjugation between the aromatic rings and nitrogen atoms and transition from the characteristic $\left(\pi-\pi^{*}\right)$ transitions of naphthalene chromophore $^{29}$. Their spectra in NMP solution showed photoluminescence emission maxima bands around (425-443) nm in the blue region. Figs. 14 and 15 shows the UV-visible absorption and photoluminescence spectra for poly(amineamide)s. Fig. 16 showed the strong fluorescence emissions inthe blue region which can be explained from the decreased intra- and intermolecular electronic interactions. The bulky, crank effective in decreasing charge transfer formation within or between polymer chains through steric hindrance. The UVvisible transmittance spectra of these poly(amine-amide)s films and cut-off wavelengths (absorption edge $\lambda_{\circ}$ ) in the range of (412-430) nm are also indicated in Fig. 16.

Electrochemical properties: The redox behaviour of poly(amine-amide)s was investigated by cyclic voltammetry for

\begin{tabular}{ccccc}
\multicolumn{5}{c}{ TABLE-4 } \\
\hline $\begin{array}{c}\text { Polymer } \\
\text { code }\end{array}$ & $\begin{array}{c}\lambda_{\text {abs,max }} \\
(\mathrm{nm})^{\mathrm{a}}\end{array}$ & $\begin{array}{c}\lambda_{\text {abs }} \\
(\mathrm{nm})^{\mathrm{a}}\end{array}$ & $\begin{array}{c}\text { Onset } \\
(\mathrm{nm})^{\mathrm{b}}\end{array}$ & $\begin{array}{c}\lambda_{\text {PL }} \lambda_{\mathrm{o}} \\
(\mathrm{nm})^{\mathrm{c}}\end{array}$ \\
\hline (pa1) & $355(352)$ & $422(420)$ & 431 & 427 \\
$(\mathrm{pb} 1)$ & $354(356)$ & $436(438)$ & 439 & 420 \\
$(\mathrm{pc} 1)$ & $350(350)$ & $418(417)$ & 425 & 418 \\
$(\mathrm{pa} 2)$ & $328(327)$ & $427(422)$ & 438 & 410 \\
$(\mathrm{pb} 2)$ & $318(319)$ & $423(419)$ & 443 & 405 \\
$(\mathrm{pc} 2)$ & $320(321)$ & $421(423)$ & 427 & 414 \\
\hline
\end{tabular}

a: UV-VIS absorption measurements in NMP $\left(1 \times 10^{-5} \mathrm{M}\right)$ at room temperature. Values in the parentheses were measured in polymer film. b: PL spectra measurements in NMP $\left(1 \times 10^{-5} \mathrm{M}\right)$. c: the cutoff wavelengths $\left(\lambda_{\mathrm{o}}\right)$ from the transmission.

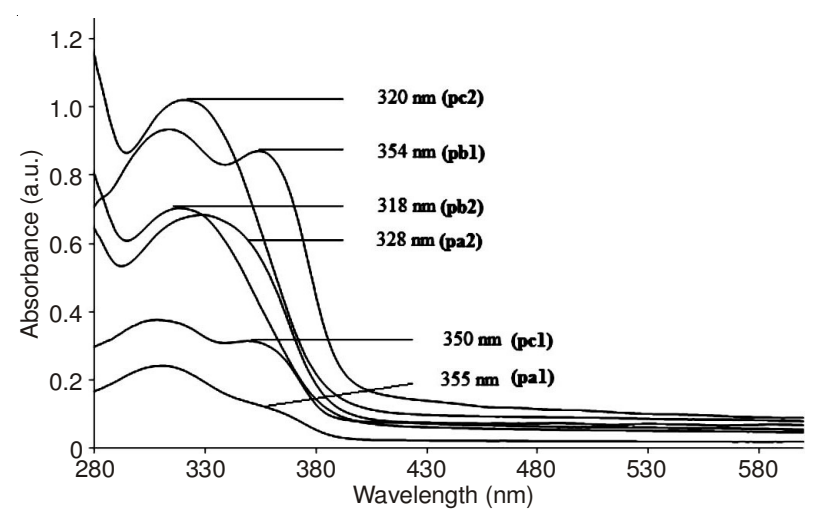

Fig. 14. UV-visible absorption spectra of poly(amine-amide)s in concentration $\left(10^{-5} \mathrm{M}\right)$ in NMP

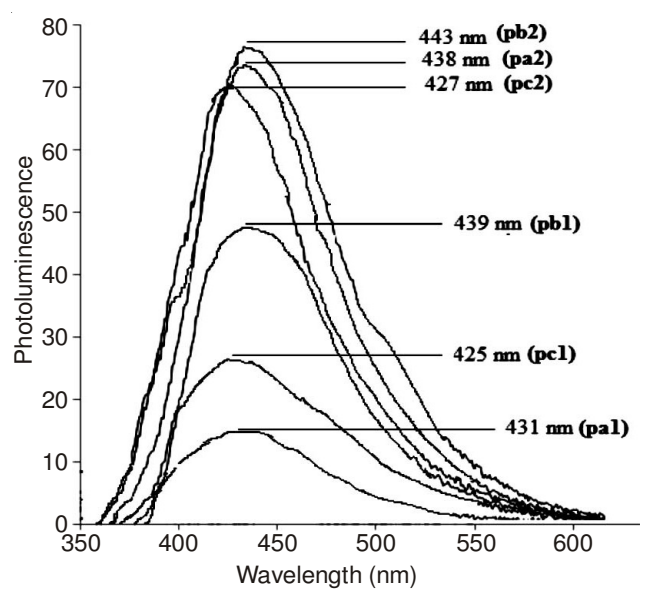

Fig. 15. UV-visible photoluminescence emission of poly(amine-amide)s in concentration $\left(10^{-5} \mathrm{M}\right)$ in NMP

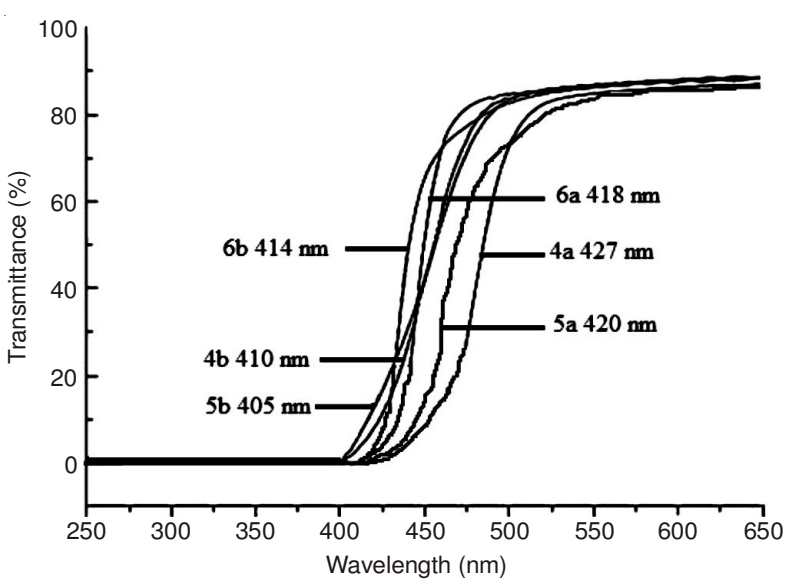

Fig. 16. UV-visible transmittance spectra of poly(amine-amide)s with a concentration $\left(10^{-5} \mathrm{M}\right)$ in NMP 


\begin{tabular}{ccccc}
\hline \multicolumn{5}{c}{ TABLE-5 } \\
\hline Polymer code & $\begin{array}{c}\mathrm{E}_{\text {onsel }} / \mathrm{V}(v s . \mathrm{Ag} / \mathrm{AgCl}) \text { in } \\
\mathrm{CH}_{3} \mathrm{CN}(\mathrm{nm})\end{array}$ & $\begin{array}{c}\lambda_{\text {abs}} \text {, onset HOMO } \\
(\mathrm{eV})\end{array}$ & $\begin{array}{c}\text { LUMO }^{\mathrm{b}} \\
(\mathrm{eV})\end{array}$ & $\begin{array}{c}\text { HOMO-LUMO gap }^{\mathrm{c}} \\
(\mathrm{eV})\end{array}$ \\
\hline pa1 & 1.35 & $422(420) 5.74$ & 2.79 & 2.95 \\
pb1 & 1.31 & $436(438) 5.70$ & 2.87 & 2.83 \\
pc1 & 1.27 & $418(417) 5.66$ & 2.69 & 2.97 \\
pa2 & 1.37 & $421(423) 5.76$ & 2.83 & 2.93 \\
pb2 & 1.34 & $423(419) 5.73$ & 2.78 & 2.95 \\
pc2 & 1.30 & $27(422) 5.69$ & 2.76 & 2.93 \\
\hline
\end{tabular}

a: The HOMO energy levels were calculated from cyclic voltammetry and were referenced to ferrocene ( $4.8 \mathrm{eV})$. b: LUMO = HOMO - gap. c: Energy gap data were calculated of thin film by the equation: Energy gap $=1240 / \lambda_{\text {onset }}$ of polymer film.

the cast film on an ITO-coated glass substrate as working electrode in dry acetonitrile containing $0.1 \mathrm{M}$ of tetrabutylammonium perchlorate as an electrolyte, under nitrogen atmosphere. All the poly(amine-amide)s showed an reversible oxidation process. Fig. 17 shows the typical cyclic voltammogram for poly(amine-amide) (pa1) recorded at scanning rate of 0.1 $\mathrm{V} / \mathrm{s}$. One reversible oxidation redox couple with half- wave at $\mathrm{E}_{1 / 2}=1.35 \mathrm{~V}$ ( vs. $\left.\mathrm{Ag} / \mathrm{Ag} / \mathrm{Cl}\right)$ in the oxidative scan was observed. Because of electrochemical stability of the films and good adhesion between the polymer and ITO substrate, the polyamide (pa1) exhibited good reversibility of electrochromic characteristics by continuous five scans between $0.0-1.60 \mathrm{~V}$ changing colour from original pale yellowish to blue. The energy of the HOMO and LUMO levels of the corresponding poly(amine-amide)s can be determined from the oxidation onset $\left(\mathrm{E}_{\text {onset }}\right)$ and the onset absorption wavelength of the UVVIS absorption of thin films ${ }^{30}$ and the results are listed in Table5. The external ferrocene/ferrocenium $(\mathrm{Fc} / \mathrm{Fc}+)$ redox standard $\mathrm{E}_{\text {onset }}$ is $0.41 \mathrm{~V}$ versus $\mathrm{Ag} / \mathrm{AgCl}$ in $\mathrm{CH}_{3} \mathrm{CN}$ and the $\mathrm{HOMO}$ energy for the $\mathrm{Fc} / \mathrm{Fc}+$ standard is $4.80 \mathrm{eV}$ with respect to the zero vacuum level. Therefore, the HOMO energy for (pa1) has been evaluated to be $5.74 \mathrm{EV}$ as follows: $\mathrm{E}_{1 / 2}$ (onset)= $\left(\mathrm{E}_{\mathrm{PC}}+\mathrm{E}_{\mathrm{pa}}\right) / 2$ where $\mathrm{pc}$ and pa are potentials of cathode and anode, respectively.

$$
\begin{aligned}
& \mathrm{E}_{1 / 2} \text { (onset) }=(1.48+1.23) / 2=1.35 \mathrm{~V} \\
& \mathrm{E}_{\mathrm{HOMO}}=\left[\mathrm{E}_{1 / 2} \text { (onset) } \% \mathrm{E}_{1 / 2}(\text { ref. })+4.8\right] \mathrm{eV} \\
& \mathrm{E}_{\mathrm{HOMO}}=[1.35 \% 0.41+4.8]=5.74 \mathrm{eV} \\
& \mathrm{Gap}=1240 / \lambda_{\text {onset }}=1240 / 420=2.95 \mathrm{eV} \\
& \mathrm{HOMO}-\mathrm{LUMO}=\mathrm{GAP} \\
& \mathrm{LUMO}=5.74 \% 2.95=2.79 \mathrm{eV}
\end{aligned}
$$

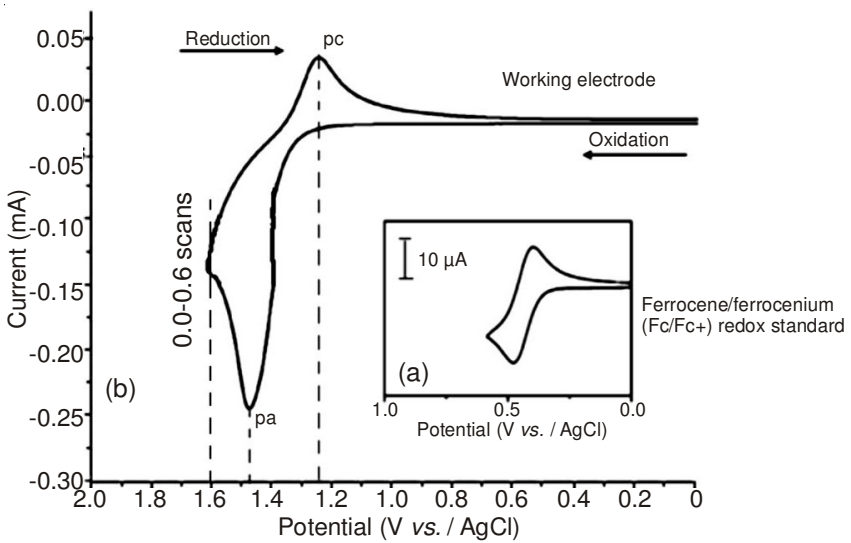

Fig. 17. Cyclic voltammograms of (a) ferrocene as reference and (b) the cast film of poly(amine-amide) (pa1) on the indium-tin oxide(ITO)coated glass substrate in $\mathrm{CH}_{3} \mathrm{CN}$ containg $0.1 \mathrm{M}$ TBAP, with a scan rate of $0.1 \mathrm{~V} / \mathrm{s}$

\section{Conclusion}

A series of new poly(amine-amide)s bearing derivedtriphenylamine units in the polymer main chain have been successfully prepared from a newly synthesized aromatic derived triphenylamine dicarboxylic acid, 4,4'-dicarboxy-4"triphenylamine with two different aromatic diamines by phosphorylation polyamidation technique. All the poly(amineamide)s were amorphous with high $\mathrm{T}_{\mathrm{g}}$ and exhibited excellent thermal stability and useful redox stability. These polymers exhibited blue photoluminescence both in film and in solution. Thus, these novel triphenylamine-containing polyamides may find applications in electroluminescent devices as hole-transporting or blue-light-emitting polymeric materials.

\section{REFERENCES}

1. C.W. Tang and S.A. Van Slyke, Appl. Phys. Lett., 51, 913 (1987).

2. C.W. Tang, S.A. Van Slyke and C.H. Chen, J. Appl. Phys., 85, 3610 (1989).

3. C. Adachi, K. Nagai and N. Tamoto, Appl. Phys. Lett., 66, 2679 (1995).

4. Y. Shirota, J. Mater. Chem., 10, 1 (2000).

5. M. Yang and Q. Zhang, J. Mater. Sci., 39, 3777 (2004).

6. A.P. Kulkarni, C.J. Tonzola, A. Babel and S.A. Jenekhe, Chem. Mater., 16, 4556 (2004).

7. M. Thelakkat, J. Hagen, D. Haarer and H.-W. Schmidt, Synth. Met., 102, 1125 (1999).

8. N.C.M. Greenham, S.C. Moratti, D.D.C. Bradley, R.H. Friend and A.B. Holmes, Nature, 365, 628 (1993).

9. M. Strukelj, F. Papadimitrakopoulos, T.M. Miller and L.J. Rothberg, Science, 267, 5206, 1969 (1995).

10. U.B. Mitschke and P. Bäuerle, J. Mater. Chem., 10, 1471 (2000).

11. E.N. Ueta, H. Nakano and Y. Shirota, Chem. Lett., 12, 2397 (1994).

12. H.H. Yang, Aromatic High-Strength Fibers, Wiley (1989).

13. Y. Imai, High Perform. Polym., 7, 337 (1995).

14. Y. Imai, React. Funct. Polym., 30, 3 (1996).

15. G.-S. Liou and S.-H. Hsiao, J. Polym. Sci. A: Polym. Chem., 40, 1781 (2002).

16. S.H. Hsiao, C.-W. Chen and G.-S. Liou, J. Polym. Sci. A: Polym. Chem., 42, 3302 (2004).

17. Y. Oishi, H. Takado, M. Yoneyama, M.-A. Kakimoto and Y. Imai, J. Polym. Sci. A: Part A: Polym. Chem., 28, 1763 (1990).

18. G.-S. Liou, S.-H. Hsiao, M. Ishida, M. Kakimoto and Y. Imai, J. Polym. Sci. Part A: Polym. Chem., 40, 2810 (2002).

19. G.-S. Liou and S.-H. Hsiao, J. Polym. Sci. Part A: Polym. Chem., 41, 94 (2003).

20. K. Ogino, A. Kanegae, R. Yamaguchi, H. Sato and J. Kurjata, Macromol. Rapid Commun., 20, 103 (1999).

21. M.-Y. Chou, M.- Leung, Y.O. Su, C.L. Chiang, C.-C. Lin, J.-H. Liu, C.-K. Kuo and C.-Y. Mou, Chem. Mater., 16, 654 (2001).

22. S.-H. Cheng, S.-H. Hsiao, T.-H. Su and G.-S. Liou, Macromolecules, 38, 307 (2005).

23. S.-H. Hsiao, Y.-M. Chang, H.-W. Chen and G.-S. Liou, J. Polym. Sci. A: Polym. Chem., 44, 4579 (2006).

24. S. Beaupre, J. Dumas and M. Leclerc, Chem. Mater., 18, 4011 (2006).

25. K. Choi, S.J. Yoo, Y.-E. Sung and R. Zentel, Chem. Mater., 18, 5823 (2006).

26. N. Yamazaki, F. Higashi and J. Kawabata, J. Polym. Sci. Polym. Chem., 12, 2149 (1974).

27. G.-S. Liou, N.-K. Huang and Y.-L. Yang, J. Polym. Sci. Part A: Polym. Chem., 44, 4095 (2006).

28. Y. Oishi, K. Mori, H. Hirahara, Y. Fujimura and K. Miya, Japan Patent, 11-255723 (1999).

29. R. Martínez-Máñez and F. Sancenón, Chem. Rev., 103, 4419 (2003).

30. D.M. de Leeuw, M.M.J. Simenon, A.R. Brown and R.E.F. Einerhand, Synth. Met., 87, 53 (1997). 